LeAndro Gustavo de Oliveira ${ }^{1}$

ANANTH KARUMANCHI ${ }^{2}$

NELSON SASS ${ }^{3}$

Revisão

Palavras-chave

Pré-eclâmpsia/fisiopatologia Linfocinas/antagonistas \& inibidores

Inflamação

Estresse oxidativo/fisiologia Endotelio vascular/fisiopatologia

Keywords

Preeclampsia/physiopathology Lymphokines/antagonists \& inhibitors Inflammation

Oxidative stress/physiology Endothelium, vascular/physiopathology

\section{Pré-eclâmpsia: estresse oxidativo, inflamação e disfunção endotelial}

\author{
Preelampsia: oxidative stress, inflammation and endothelial \\ dysfunction
}

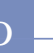

\section{Resumo}

Pré-eclâmpsia é uma síndrome sistêmica caracterizada por intenso estado inflamatório e antiangiogênico. A fisiopatologia da pré-clâmpsia envolve alterações no processo de invasão trofoblástica, com consequente inadequado suprimento sanguíneo uterino e estresse oxidativo do tecido placentário. As alterações placentárias decorrentes desse processo levam à maior produção de sFlt-1, um receptor solúvel para as moléculas de VEGF e PIGF. O sFlt- 1 impede com que VEGF e PIGF realizem suas funções na homeostase endotelial, culminando com disfunção dessas células. De uma maneira geral, os processos inflamatórios, de disfunção endotelial e estresse oxidativo estão interligados e agem de maneira sinérgica. Trabalhos recentes têm demonstrado que elevações nas concentrações séricas de sFlt-1 ocorrem 5 a 6 semanas antes das manifestações clínicas da pré-eclâmpsia. Concomitantemente, observa-se queda nas concentrações séricas de PlGF. Sendo assim, as dosagens séricas de sFlt-1 e PlGF têm sido sugeridas para o diagnóstico precoce de pré-eclâmpsia. Ademais, os conhecimentos adquiridos a respeito dos fatores antiangiogênicos proporcionam ainda a possibilidade de novas linhas de pesquisa sobre possíveis terapias para a pré-eclâmpsia. Neste artigo, foram revisados os aspectos inflamatórios e antiangiogênicos envolvidos na fisiopatologia da pré-eclâmpsia. Por fim, foram correlacionados esses aspectos com o risco elevado para doenças cardiovasculares apresentado por essas pacientes ao longo de suas vidas.

\section{Abstract}

Preeclampsia is a systemic syndrome characterized by inflammatory and antiangiogenic states. The pathogenesis of preeclampsia involves deficient trophoblast invasion that is responsible for altered uterine blood flow and placental oxidative stress. The damaged placenta produces higher concentrations of sFlt-1, a soluble receptor for VEGF and PIGF that is released in the maternal circulation and is involved in endothelial dysfunction. Actually, all processes involved in inflammation, endothelial dysfunction and oxidative stress are strongly correlated and act in a synergistic way. Recent data have shown that an increase in serum concentrations of sFlt- 1 initiates 5 to 6 weeks before the clinical manifestations of preeclampsia and these alterations correlate with a decrease in serum concentrations of PIGF. Therefore, both sFlt-1 and PIGF have been suggested to be useful for an early-diagnosis of preeclampsia. The knowledge about the role of antiangiogenic factors in the pathogenesis of preeclampsia has raised the possibility of a therapy involving these factors. In this article we revisited the pathogenesis of preeclampsia addressing its antiangiogenic and inflammatory states. In conclusion, we correlated these alterations with the higher risk for cardiovascular diseases presented by these women in future life.

Correspondência:

Leandro Gustavo de Oliveira Rua Napoleão de Barros, 875 - Vila Clementino (EP: 04023-900 - São Paulo (SP), Brasil E-mail: leminas@ig.com.br

Recebido

$24 / 8 / 10$

Aceito com modificacōoes

$16 / 11 / 10$
' Pesquisador Associado do Departamento de Obstetrícia e do Laboratório de Imunologia Clínica e Experimental da Disciplina de Nefrologia da Universidade Federal de São Paulo - UNIFESP -, São Paulo (SP), Brasil; Responsável pelo Laboratório de Investigação Clínica e Experimental do Hospital e Maternidade Escola "Vila Nova Cachoeirinha" - São Paulo (SP), Brasil.

2 Professor Associado do Departamento de Medicina da Universidade de Harvard - USA.

${ }^{3}$ Professor Livre-docente do Departamento de Obstetrícia da Universidade Federal de São Paulo - UNIFESP -, São Paulo (SP), Brasil; Chefe de Clínica Obstétrica do Hospital e Maternidade Escola "Vila Nova Cachoeirinha". 


\section{Introdução}

A pré-eclâmpsia é uma doença de distribuição mundial que atinge 5 a $8 \%$ das gestantes ${ }^{1}$. Seu diagnóstico é feito a partir da $20^{a}$ semana de gravidez ou nos primeiros dias após o parto e baseia-se no desenvolvimento de hipertensão arterial (PA $\geq 140 \times 90 \mathrm{mmHg}$ ) e proteinúria $(\geq 300 \mathrm{mg} / 24 \text { horas })^{2}$. A maioria dos diagnósticos é feita no terceiro trimestre de gravidez, sendo que a gravidade da doença é maior quanto mais precocemente se dá sua manifestação clínica.

A pré-eclâmpsia é uma doença sistêmica caracterizada por intensa resposta inflamatória, lesão endotelial, agregação plaquetária, ativação do sistema de coagulação e aumento da resistência vascular generalizada ${ }^{3,4}$. Sendo assim, todos os órgãos podem sofrer efeitos da pré-eclâmpsia. Nos rins, é possível encontrar a princípio uma lesão glomerular típica, a glomeruloendoteliose. No entanto, algumas pacientes apresentam outros tipos de comprometimento, como é o caso da glomeruloesclerose segmentar focal ${ }^{5}$. Em quadros graves, pode ainda haver progressão para graus variados de injúria renal, como o que se vê nos casos de síndrome hemolítico-urêmica. Nos pulmões, o comprometimento endotelial e consequente permeabilidade vascular exagerada podem culminar com o edema pulmonar. $\mathrm{O}$ dano vascular hepático, associado ao consumo exagerado de plaquetas e a hemólise sistêmica, caracterizam o que se denomina "síndrome HELLP" (do inglês hemolysis, elevated liver enzymes and low platelets), quadro clínico que se associa a taxas elevadas de morbimortalidade, tanto maternas quanto perinatais. A lesão endotelial cerebral leva ao edema difuso e ao quadro dramático de eclâmpsia, caracterizada por convulsões tônico-clônicas nessas pacientes. Fenômenos hemorrágicos e áreas de infartos também podem ser vistos no tecido cerebral ${ }^{6,7}$. Uma alteração cada vez mais descrita em pacientes com préeclâmpsia é a leucoencefalopatia posterior, que se caracteriza por edema e infartos que acometem a substância branca cerebral, principalmente no lobo occipital e em porções posteriores dos lobos parietais ${ }^{8}$. Essa lesão é também vista em pacientes em uso de drogas antiangiogênicas para o tratamento de alguns tipos de neoplasias. A ocorrência de lesão similar nas duas morbidades sugere a presença de fatores antiangiogênicos endógenos participando da fisiopatologia da pré-eclâmpsia ${ }^{9}$.

O impacto da pré-eclâmpsia sobre a gestação é visto como uma preocupação mundial, justamente por representar importante causa de morbimortalidade materna e perinatal. Apesar de esses números certamente serem subestimados, calcula-se que cerca de 76.000 mortes maternas e 500.000 mortes perinatais são relacionadas à pré-eclâmpsia todos os anos ${ }^{10}$. A partir de dados do Sistema Único de Saúde (SUS) brasileiro, é possível afirmar que, no Brasil, cerca de três gestantes morrem por dia vítimas das complicações causadas pela pré-eclâmpsia ${ }^{11}$. É comum se ouvir que a eclâmpsia representa o estágio final da doença. Mas é preciso ter em mente que "as mulheres morrem muito mais por pré-eclâmpsia do que por eclâmpsia”. Essa afirmação salienta a importância do diagnóstico precoce e adequada condução dos casos de pré-eclâmpsia desde o início de sua manifestação e, até mesmo, antes da instalação de sua forma clínica - o que seria o ideal.

\section{Etiopatogenia}

\section{A placenta}

Apesar de sua elevada importância, a pré-eclâmpsia ainda não tem causas bem estabelecidas. No entanto, sabese que a placenta é peça fundamental para sua ocorrência, não havendo necessidade nem mesmo do desenvolvimento fetal. Tal constatação se deu devido ao fato de que casos de mola hidatiforme completa, situações em que o feto não se desenvolve, podem ser acompanhados de pré-eclâmpsia grave. Ademais, gestações que se desenvolvem fora da cavidade uterina (gestações ectópicas com evolução além da $20^{\mathrm{a}}$ semana) também podem ser complicadas por pré-eclâmpsia, mostrando que o útero também não se faz necessário. Em conclusão, sabe-se que a única cura para a pré-eclâmpsia é o parto, quando a placenta é por fim removida.

Dentro de sua etiopatogenia, o desenvolvimento da pré-eclâmpsia pode ser dividido em três fases. Para o melhor entendimento dessas fases é preciso lembrar que o feto representa um semienxerto para o organismo materno devido à presença da carga genética paterna, mas é a placenta que entra em contato direto com o sangue materno e deve, assim, ser tolerada por seu sistema imune. Sendo assim, é possível dizer que a primeira fase da pré-eclâmpsia é representada por uma quebra de tolerância, na qual a interação do trofoblasto com os leucócitos deciduais gera, entre outras alterações, inadequada produção de citocinas e quimiocinas que tornam a interface materno-fetal imprópria para o desenvolvimento normal da gravidez ${ }^{12}$.

Seguindo a quebra de tolerância e ainda no primeiro trimestre de gestação, inicia-se a segunda fase de desenvolvimento da doença, quando o trofoblasto extraviloso (TEV) não desempenha normalmente suas funções e não invade adequadamente o terço interno da parede uteri$\mathrm{na}^{13}$. No fenômeno da invasão trofoblástica normal, o TEV atinge as arteríolas espiraladas miometriais já em torno de 6 a 8 semanas de gravidez, promovendo, nesse momento, verdadeira obstrução do fluxo sanguíneo que banha os espaços intervilosos. Essa forma de desenvolvimento permite que a gravidez se desenvolva inicialmente em um ambiente hipóxico, protegendo as células fetais do excessivo aporte de $\mathrm{O}_{2}$, fato discutido mais adiante ${ }^{14}$. A medida que a gestação progride, o TEV promove um 
desarranjo da camada muscular das arteríolas espiraladas uterinas e, assumindo ainda características de células endoteliais, substitui a camada endotelial desses vasos (Figura 1). Quando todo esse processo se dá de forma e em momento adequados, ocorre o relaxamento e maior complacência das artérias uterinas que se tornam capazes de fornecer adequado suprimento sanguíneo útero-placentário ao longo da gravidez ${ }^{15}$.

Não se sabe ao certo a razão pela qual o trofoblasto não consegue desempenhar adequadamente suas funções de invasão e remodelamento dos vasos uterinos. Mas é indiscutível que alterações imunológicas são determinantes nesse sentido ${ }^{13,16-18}$. As células trofoblásticas apresentam características especiais quanto à expressão de moléculas do sistema HLA (do inglês Human Leukocitary Antigen). Diferentemente da maioria das células do organismo, o trofoblasto expressa apenas moléculas HLA-G, HLA-C e HLA-E ${ }^{19,20,21}$. Interações entre essas moléculas e populações distintas de leucócitos na interface materno-fetal, em especial com células natural killers uterinas (uNK) e macrófagos, devem ocorrer de forma harmônica para que seja criado um ambiente favorável à tolerância imunológica. Há ainda fenômenos de tolerância periférica, em que atuam células regulatórias que agem modulando a atividade de linfócitos T durante a gravidez. Em trabalho recente, demonstrou-se a redução de células NKT periféricas em gestantes normais ${ }^{22}$. Essas células são importantes produtoras de citocinas e seu adequado equilíbrio pode ser fundamental durante a gestação. Saito et al. ${ }^{23}$ demonstraram a redução na porcentagem de células $T$ reguladoras em pacientes com pré-eclâmpsia, o que levaria a maior ativação de linfócitos e consequentemente a maior resposta inflamatória. Pode-se dizer, então, que a quebra de tolerância materno-fetal ou materno-placentária pode levar à placentação deficiente, resposta inflamatória e, consequentemente, à pré-eclâmpsia.

\section{Estresse oxidativo e resposta inflamatória}

A invasão trofoblástica deficiente implica mau controle da oxigenação do espaço interviloso na fase inicial da gravidez e na persistência das características primárias das artérias uterinas espiraladas, que mantêm sua elevada resistência. Nesses casos, o sangue banha as vilosidades coriônicas na forma de "jatos intermitentes" e de alta pressão. Assim, o fluxo sanguíneo útero-placentário simula o que acontece em casos de lesão por isquemiareperfusão $(\mathrm{I} / \mathrm{R})^{24,25}$. Entre outras alterações, esse tipo de injúria é marcado por produção exagerada de ROS (do inglês reactive oxigen species) e RNS (do inglês reactive nitrogen species) sempre que as moléculas de oxigênio são reintroduzidas no tecido após o momento isquêmico. Tanto ROS quanto RNS constituem moléculas de radicais livres geradas principalmente na mitocôndria celular. A produção de ROS é caracterizada pela geração de moléculas superóxido $\left(\mathrm{O}_{2} \bullet^{-}\right)$, que, em situações de ausente ou insuficiente capacidade tecidual antioxidativa, desencadeiam uma cascata de eventos com maior produção de ROS e peroxidação lipídica (Figura 2). Todas essas moléculas levam à resposta inflamatória e dano celular ${ }^{26}$.

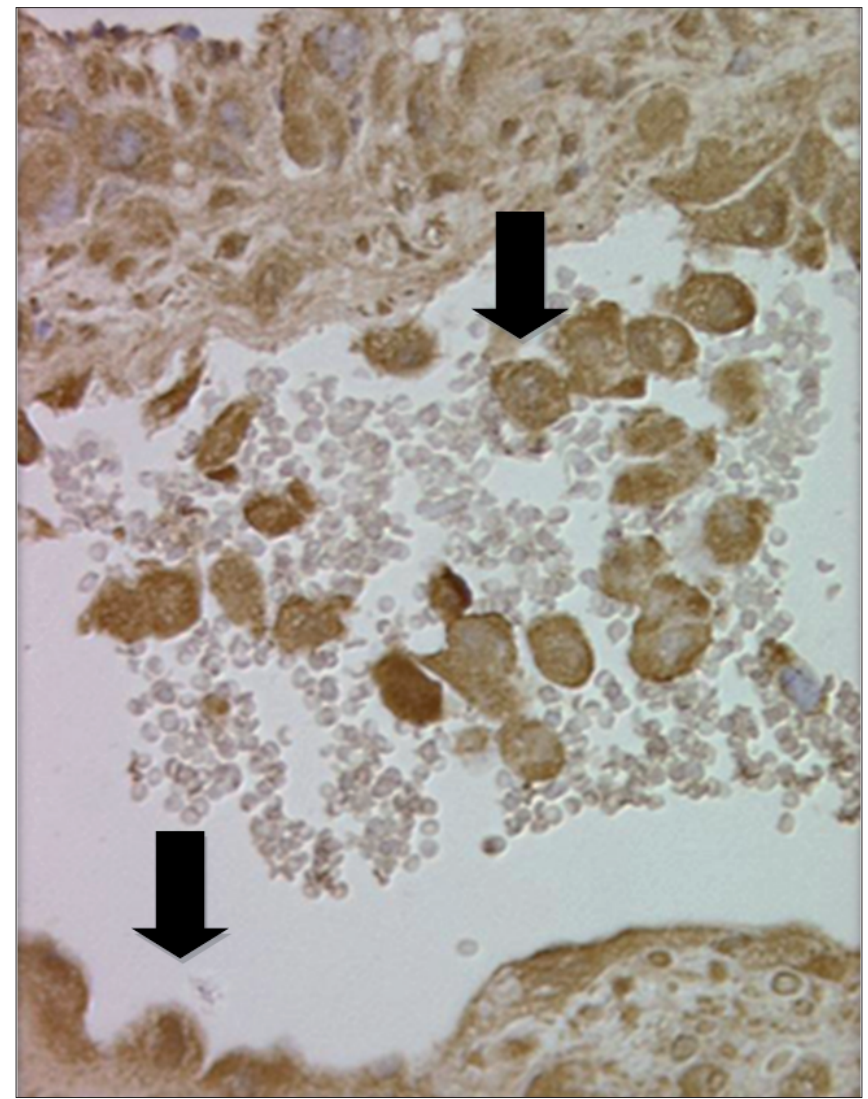

Figura 1 - Secção seriada de biópsia de leito placentário no qual se observa uma arteríola espiralada. Notam-se os trofoblastos extravilosos presentes na luz do vaso espiralado e substituindo as células endoteliais (setas).

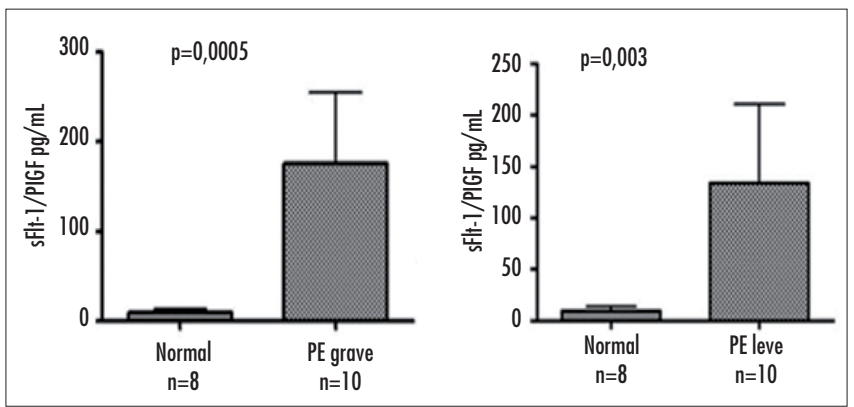

Figura 2 - Representação esquemática do processo de estresse oxidativo provocado pelo fenômeno de isquemia-reperfusão (Modificado de Cindrova-Davies ${ }^{25}$. Os momentos de reintrodução da oxigenação provocam aumento abruto das moléculas de $\mathrm{O}_{2}$ e geração de $\mathrm{ROS}$. As enzimas $\mathrm{Cu} / \mathrm{Zn}$ ou Mn superóxido dismutase participam como enzimas antioxidantes promovendo a formação de $\mathrm{H}_{2} \mathrm{O}_{2}$, enquanto catalase e glutatione peroxidase seguem na formação de $\mathrm{H}_{2} \mathrm{O}$, que é liberada no organismo. Na ausência ou insuficiência do mecanismo antioxidativo, ocorre um desvio nessa reação com maior formação de ROS, peroxidação lipídica e lesão celular. 
O tecido placentário, principalmente o sinciciotrofoblasto, possui pouca capacidade antioxidativa. Diante disso, as alterações na maneira com que o sangue perfunde o espaço interviloso provocam o desenvolvimento do que se caracteriza como estresse oxidativo. O sincício então passa a apresentar maior atividade de apoptose/necrose, liberando, na circulação materna, grande quantidade de material sincicial, fatores antiangiogênicos e debris. Todas essas moléculas provocam ativação de leucócitos sistêmicos, geram estímulos para maior adesão plaquetária, vasoconstrição e resposta inflamatória generalizada. Essa resposta inflamatória é mediada principalmente por citocinas pró-inflamatórias como TNF (fator de necrose tumoral), INF-gama (interferon-gama) e IL-6 (interleucina$6)^{26}$. Knight et al. ${ }^{27}$ demonstraram a presença de maior quantidade de micropartículas sinciciais na circulação de pacientes com pré-eclâmpsia do que em gestantes normais. Recentemente, Germain et al. ${ }^{28}$ demonstraram que essas partículas derivadas do processo necrótico sincicial estimulam a produção de citocinas inflamatórias por monócitos circulantes, participando ativamente do desencadeamento e da manutenção da resposta inflamatória na pré-eclâmpsia. Outros trabalhos também apontam a elevação de marcadores de resposta inflamatória de fase aguda na pré-eclâmpsia, como leucocitose, hipoalbuminemia, elevação de proteína C-reativa ${ }^{29,30}$. Borzychowski et al. ${ }^{31}$ resumiram o processo inflamatório da pré-eclâmpsia salientando que o ápice desse processo determina o aparecimento do quadro clínico da doença.

\section{Disfunção endotelial}

Os primeiros relatos nas décadas de 1980 e 1990 ressaltaram as disfunções endoteliais como base das alterações relacionadas à pré-eclâmpsia ${ }^{32,33}$. Características como elevada permeabilidade vascular, presença sérica de fatores relacionados à injúria endotelial como fibronectina, fator VIII e trombomodulina, além da elevada reatividade vascular na pré-eclâmpsia, corroboram as hipóteses desses autores. As micropartículas sinciciais que atuam no desenvolvimento da resposta inflamatória da pré-eclâmpsia também são capazes de lesar diretamente o endotélio e promover ativação de neutrófilos ${ }^{34}$. Cockell et al. ${ }^{35}$ demonstraram a agressão endotelial provocada por essas partículas em modelo experimental. Esses autores demonstraram essa alteração colocando as micropartículas sinciciais em contato com vasos do omento materno.

Os principais fatores angiogênicos que desempenham papéis fundamentais na manutenção da homeostase endotelial são VEGF (do inglês vasculo-endothelial growth), PlGF (do inglês placental growth factor) e TGF- $\beta 1$ (do inglês tissue growth factor) ${ }^{36,37}$. O VEGF participa da manutenção endotelial de órgãos como rim, fígado e cérebro, sinalizando por meio de dois receptores, FlK e
Flt-1. Este último também tem como ligante o $\mathrm{PlGF}^{6}$. $O$ receptor para TGF- $\beta 1$ é constituído por um complexo formado por Alk5-T $\beta$ RII-Endoglin. O equilíbrio na produção de fatores angiogênicos é também importante para o próprio desenvolvimento placentário, caso contrário, o estímulo exacerbado determinado pelos fatores angiogênicos produzidos pelo trofoblasto e células da interface materno-fetal transformaria a placenta em um grande hemangioma.

O estresse oxidativo leva à produção placentária de grande quantidade de fatores antiangiogênicos como o sFlt-1 (do inglês soluble fms-like tyrosin) e a sEndoglin (do inglês soluble endoglin). O sFlt-1 é um receptor solúvel que se forma por alternative splicing, levando à perda da porção transmembrana do Flt-1. Dessa forma, o sFlt-1 se liga às moléculas de VEGF e PlGF circulantes e impede com que esses fatores angiogênicos se liguem aos seus receptores comuns na membrana celular (Figura 3$)^{6}$. Maynard et al. ${ }^{38}$ demonstraram que a injeção de sFlt-1 em camundongos prenhes determina hipertensão arterial e proteinúria nesses animais, simulando a expressão clínica da pré-eclâmpsia. Outros trabalhos mostram que o VEGF é responsável pela manutenção das fenestras do endotélio glomerular e que a presença do sFlt-1 impede a função do VEGF no glomérulo e leva à glomeruloendoteliose e proteinúria $^{39}$. A sEndoglin age de forma semelhante ao sFlt-1, impedindo a ação angiogênica do seu ligante, o TGF- $\beta 1$.

Já foi demonstrada a presença de elevadas concentrações de sFlt-1 no sangue de pacientes com pré-eclâmpsia. Esses autores demonstraram que elevações nos níveis séricos de sFlt-1 podem ser identificadas cerca de 5 semanas antes das manifestações clínicas da pré-eclâmpsia, sugerindo que sua dosagem poderia ser utilizada então para o diagnóstico precoce da doença ${ }^{40}$. Associados à elevação dos níveis de sFlt-1, os autores demonstraram ainda a redução dos níveis de VEGF e PIGF livres na circulação materna, decorrente de suas ligações aos receptores solúveis. Romero et al. ${ }^{41}$ demonstraram também que as elevações plasmáticas de sFlt-1 e s-Endoglin associadas à redução de PlGF, precedem as manifestações clínicas da pré-eclâmpsia em cerca de 5 semanas.

Recentemente, foi avaliada a relação entre as concentrações séricas de sFlt-1 e PlGF em pacientes com pré-eclâmpsia de instalação precoce ( $<34$ semanas) por um método automatizado de eletroquimioluminescencia. A média dessa relação foi 175,8 entre as pacientes com pré-eclâmpsia grave e 134,1 entre as pacientes com préeclâmpsia leve. No grupo controle esta relação foi de 9.7 $(\mathrm{p}=0,0005$ e $\mathrm{p}=0,003$, respectivamente) (Figura 4).

O papel dos fatores antiangiogênicos na disfunção endotelial observada na pré-eclâmpsia tem sido extensamente avaliado, sendo que o seu conhecimento representa 

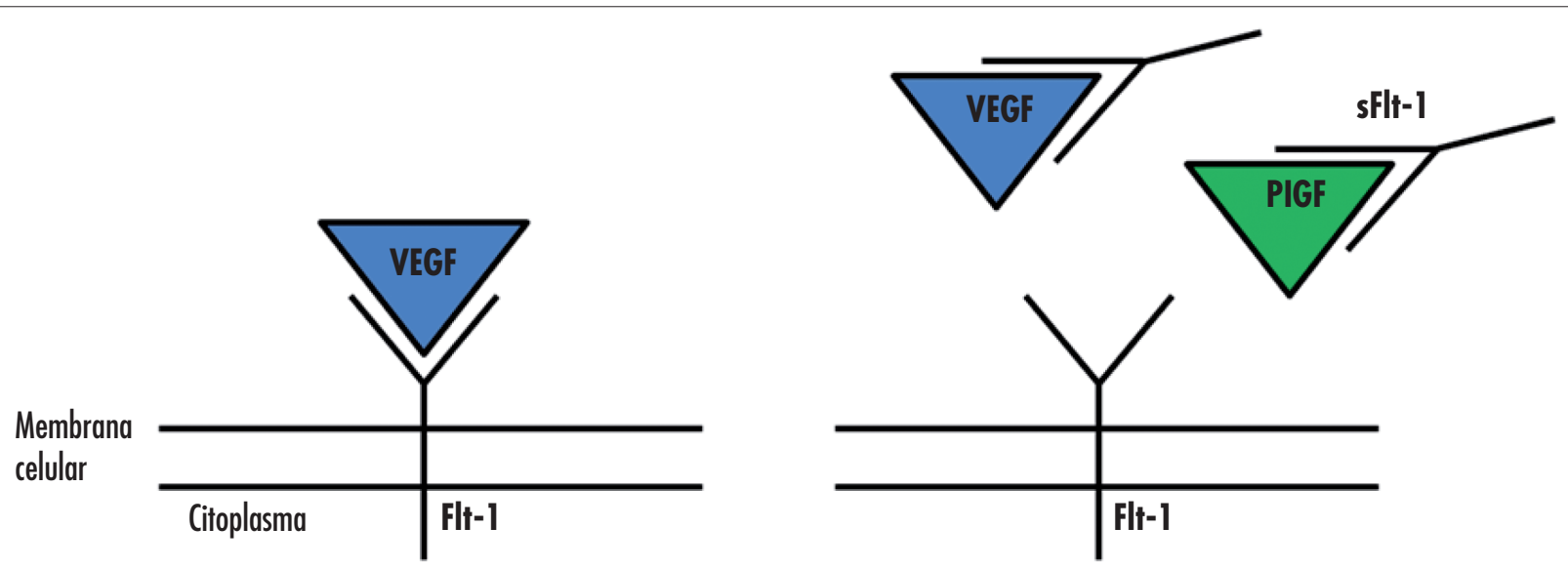

Figura 3 - No primeiro desenho observa-se a ligação normal entre o VEGF e seu receptor na membrana celular. Em seguida, observa-se a interceptação dos fatores angiogênicos pelo sFlt-1, impedindo sua ação celular.

interesse tanto na avaliação do diagnóstico precoce da doença quanto no potencial para possíveis formas terapêuticas para a pré-eclâmpsia.

\section{Obesidade e pré-eclâmpsia}

Diversos trabalhos apontam a obesidade como fator de predisposição para pré-eclâmpsia ${ }^{42}$. Em revisão sistemática, Duckitt e Harrington ${ }^{43}$ demonstraram que em mulheres com índice de massa corporal (IMC) $\geq 30$, o risco relativo para desenvolver pré-eclâmpsia é igual a 2,1. Bianco et al. ${ }^{44}$ demonstraram que a chance de pacientes com IMC $\geq 35$ desenvolverem pré-eclâmpsia é superior a quatro vezes em relação à população saudável. Contrariamente, mulheres com IMC $<20$ possuem menores chances de desenvolver a doença.

Os fatores responsáveis por esse aumento não são totalmente elucidados, mas a síndrome metabólica que acompanha o quadro de obesidade apresenta-se como fator importante. Nesse contexto, o tecido adiposo é responsável por desencadear resposta inflamatória sistêmica crônica, elevada resistência a insulina, hiperlipidemia e disfunção endotelial ${ }^{31}$. A presença de adipocinas inflamatórias como TNF- $\alpha$, IL-6 e MCP-1 são características da obesidade. Sabe-se ainda que, a obesidade é diretamente relacionada ao estresse oxidativo. $\mathrm{O}$ acúmulo intracelular de triglicérides causa disfunção mitocondrial, com maior transporte de elétrons e geração de $\operatorname{ROS}^{45}$. Sendo assim, o efeito danoso dos lipídeos sobre o endotélio se dá tanto por ação direta quanto por geração de $\mathrm{ROS}^{46}$. O excesso de lipídeos tóxicos (por exemplo, palmitato e LDL oxidada) no retículo endoplasmático de macrófagos contribui para o desenvolvimento de ROS e aterosclerose ${ }^{47}$. Na gravidez, a lipotoxicidade reduz a capacidade de invasão trofoblástica, causa disfunção endotelial tanto sistêmica materna quanto placentária e leva às alterações metabólicas e funcionais placentárias ${ }^{46}$. Todas essas alterações estão relacionadas à

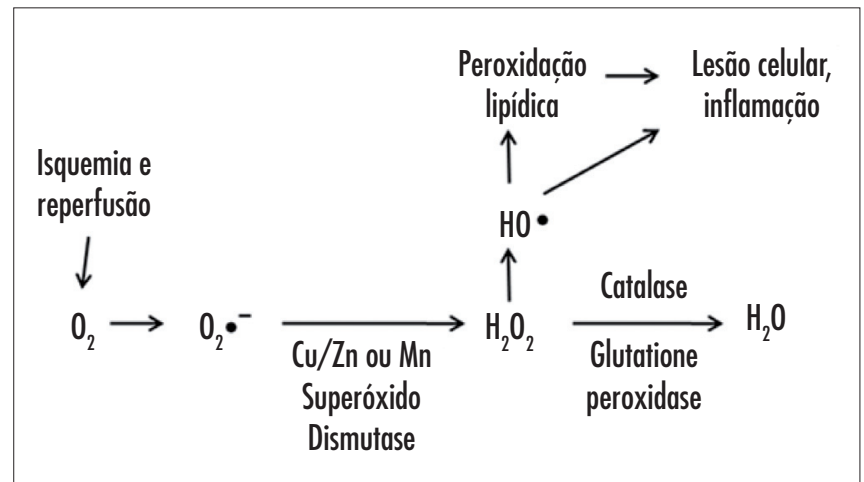

Figura 4 - Avaliação da relação sFlt-1/PIGF em pacientes com préeclâmpsia grave e leve (resultados preliminares).

fisiopatologia da pré-eclâmpsia. Diante disso, supõe-se que os fatores antiangiogênicos, principalmente o sFlt-1, possam também ser produzidos de maneira exacerbada em mulheres obesas. Esse aspecto ainda não está elucidado.

\section{Repercussões da pré-eclâmpsia sobre os recém-} nascidos

A pré-eclâmpsia representa uma das mais importantes causas de morbimortalidade perinatal em todo o mundo ${ }^{1}$. Nesse grupo de pacientes, é grande a incidência de restrição de crescimento fetal, recémnascidos prematuros e asfixiados ${ }^{10}$. A fisiopatologia de todas essas alterações é, na maioria das vezes, atribuída à insuficiência placentária crônica. Entretanto, pouco se sabe sobre a complexidade dos fatores responsáveis por problemas tanto perinatais quanto ao longo da vida dos filhos de mães com pré-eclâmpsia. Da mesma forma que ocorre nos casos de doenças maternas como obesidade e diabetes, em que parece existir certa "programação" para o desenvolvimento de doenças na vida adulta dos filhos dessas pacientes ${ }^{48}$, acredita-se que a pré-eclâmpsia possa determinar o futuro desses indivíduos. Byers et al. ${ }^{49}$ 
demonstraram recentemente, em modelo experimental de pré-eclâmpsia (pré-eclâmpsia-like), que camundongos transfectados com vetores carreando sFlt-1 deram à luz proles apresentando importantes alterações vasculares, e que essas alterações se expressavam até a vida adulta dessas crias. Sendo assim, é possível supor que as alterações vasculares e inflamatórias observadas em mães com pré-eclâmpsia tenham repercussões sobre os seus conceptos e essas se expressem tanto no momento perinatal quanto na vida adulta.

Amarilyo et al. ${ }^{50}$ demonstraram recentemente em sangue de cordão umbilical de recém-nascidos pequenos para a idade gestacional, elevadas concentrações tanto de citocinas inflamatórias, como TNF- $\alpha$ e IL- 6 , quanto de proteína C-reativa. De maneira interessante, esses autores não encontraram correlação entre níveis de PH no sangue de cordão umbilical e as concentrações desses marcadores inflamatórios. Conceitos recentes sobre o comprometimento cerebral resultante da hipóxia fetal crônica apontam para importante papel do estresse oxidativo e produção de citocinas e quimiocinas inflamatórias no desenvolvimento desde alterações leves até casos graves, como a paralisia cerebral. Esses conceitos resultam no que se caracteriza hoje como FIRS (do inglês: fetal inflammatory response syndrome $)^{51,52}$. De maneira interessante, Guo et al..$^{53}$ demonstraram em trabalho experimental com guinea pigs que a resposta inflamatória não é proporcional à privação de oxigênio. Ou seja, a hipóxia crônica pode desencadear a resposta inflamatória, mas o real comprometimento resultante depende de uma má regulação dessa resposta. Dessa forma, acredita-se que as alterações inflamatórias e antiangiogênicas maternas encontradas na pré-eclâmpsia possam contribuir diretamente para o comprometimento dos recém-nascidos dessas pacientes, bem como para com problemas que aparecerão na vida adulta dos mesmos. A pré-eclâmpsia constitui uma das questões mais fascinantes da Obstetrícia, pois resulta de anomalias na interface materno-fetal que se multiplicam em nível sistêmico materno. Os conhecimentos atuais sobre a pré-eclâmpsia permitem estabelecer três conceitos fundamentais:
1. ainda que seja identificada clinicamente após a $20^{a}$ semana, pode-se dizer que a pré-eclâmpsia trata-se, na realidade de doença crônica, uma vez que seu início ocorre nas fases iniciais da gravidez e da invasão trofoblástica;

2. a doença tem como característica o acometimento materno generalizado, pois os estados inflamatórios e antiangiogênicos atingem todos os órgãos e sistemas; o comprometimento materno não se limita aos aspectos clínicos "agudos" instalados durante a gestação, mas resulta em danos sistêmicos permanentes, responsáveis por manifestações clínicas tardias como doenças cardiovasculares e renais ${ }^{54}$. Dessa forma, é sabido que mulheres com histórico de pré-clâmpsia têm menor expectativa de vida quando comparadas àquelas que tiveram gestações sem intercorrências. Essa nova perspectiva nos leva para um novo pensamento a ser difundido: "uma vez pré-eclâmpsia, sempre pré-eclâmpsia”.

Apesar de ste texto ter sido iniciado no que se chama hoje de pré-eclâmpsia placentária, em muitas situações clínicas não conseguimos encontrar na placenta grandes alterações que justifiquem as repercussões sistêmicas maternas. Ou seja, nos deparamos com uma paciente com quadro grave de pré-eclâmpsia, cujo feto apresenta peso e líquido amniótico dentro da normalidade e vitalidade fetal preservada. É o que chamamos, então, de pré-eclâmpsia materna, em que se acredita que uma resposta inflamatória crônica basal já exista e que esta passa a ser exacerbada durante a gestação ${ }^{4}$. São os grandes exemplos desse quadro o diabetes mellitus e a obesidade.

Os conhecimentos atuais, em especial os vinculados às anomalias na angiogênese placentária e sistêmica, podem abrir novas perspectivas quanto à pré-eclâmpsia, promovendo possibilidades terapêuticas que possam agir interceptando ou mesmo anulando a ação de fatores antiangiogênicos, trazendo assim melhores prognósticos tanto maternos quanto perinatais. Espera-se que os conceitos discutidos aqui sejam úteis para auxiliar na construção de uma nova visão do problema "pré-eclâmpsia" e encorajar jovens pesquisadores.

\section{Referências}

1. ACOG Committee on Obstetric Practice. ACOG practice bulletin. Diagnosis and management of preeclampsia and eclampsia. Number 33, January 2002. American College of Obstetricians and Gynecologists. Int J Gynaecol Obstet. 2002;77(1):67-75.

2. Report of the National High Blood Pressure Education Program Working Group on High Blood Pressure in Pregnancy. Am J Obstet Gynecol. 2000; 183(1):S1-22.

3. Roberts JM. Endothelial dysfunction in preeclampsia. Semin Reprod Endocrinol. 1998; 16(1):5-15.
4. Borzychowski AM, Sargent IL, Redman CW. Inflammation and preeclampsia. Semin Fetal Neonatal Med. 2006;1 11(5):309-16.

5. Oliveira LG, Sass N, Camano L, Moura LAR, Bergamin CS. Alterações glomerulares na pré-eclâmpsia: uma revisão histórica. Femina. 2004;32(10):865-9.

6. Wang A, Rana S, Karumanchi SA. Preeclampsia: the role of angiogenic factors in its pathogenesis. Physiology (Bethesda). $2009 ; 24(3): 147-58$ 
7. Young BC, Levine RJ, Karumanchi SA. Pathogenesis of preeclampsia. Annu Rev Pathol. 2010;5:173-92.

8. Sibai BM. Diagnosis, prevention, and management of eclampsia. Obstet Gynecol. 2005; 105(2):402-10.

9. Hinchey J, Chaves C, Appignani B, Breen J, Pao L, Wang A, et al. A reversible posterior leukoencephalopathy syndrome. N Engl J Med. 1996;334(8):494-500.

10. Duley L. The global impact of pre-eclampsia and eclampsia. Semin Perinatol. 2009;33(3):130-7.

11. Brasil. Ministério da Saúde. DATASUS [Internet]. Informações de Saúde. Estatísticas vitais. Mortalidade e nascidos vivos: óbitos maternos. 2008 [citado 2010 Jan 10]. Disponível em: http:// www2.datasus.gov.br/DATASUS/index.php? area=0205

12. Moffett $A$, Hiby SE. How does the maternal immune system contribute to the development of pre-eclampsia? Placenta. 2007;28 Suppl A:S51-6.

13. Lash GE, Otun HA, Innes BA, Kirkley M, De Oliveira L, Searle RF, et al. Interferon- $\gamma$ inhibits extravillous trophoblast cell invasion by a mechanism that involves both changes in apoptosis and protease levels. FASEB J. 2006;20(14):2512-8.

14. Red-Horse K, Zhou Y, Genbacev O, Prakobphol A, Foulk R, McMaster $M$, et al. Trophoblast differentiation during embryo implantation and formation of the maternal-fetal interface. J Clin Invest. 2004; 114(6):744-54

15. De Oliveira LG, Lash GE, Murray-Dunning C, Bulmer JN, Innes BA, Searle RF, et al. Role of interleukin 8 in uterine natural killer cell regulation of extravillous trophoblast cell invasion. Placenta. 2010;31 (7):595-601

16. Moffett-King A. Natural killer cells and pregnancy. Nat Rev Immunol. 2002;2(9):656-63.

17. Saito $S$, Nakashima A, Shima T, Ito M. Th $1 / T h 2 / T h 17$ and regulatory T-cell paradigm in pregnancy. Am J Reprod Immunol. 2010;63(6):601-10.

18. Parham P. NK cells and trophoblasts: partners in pregnancy. J Exp Med. 2004;200(8):951-5.

19. Apps R, Gardner L, Hiby SE, Sharkey AM, Moffett A. Conformation of human leucocyte antigen- $C$ molecules at the surface of human trophoblast cells. Immunology. 2008;124(3):322-8.

20. Apps R, Murphy SP, Fernando R, Gardner L, Ahad T, Moffett A. Human leucocyte antigen (HLA) expression of primary trophoblast cells and placental cell lines, determined using single antigen beads to characterize allotype specificities of anti-HLA antibodies. Immunology. 2009; 127(1):26-39.

21. Trowsdale J, Moffett A. NK receptor interactions with MHC class I molecules in pregnancy. Semin Immunol. 2008;20(6):317-20.

22. De Oliveira L, Larocca R, Sass N, Câmara NO. Proportion of invariant NKT cells in Preeclampsia. J Reprod Immunol. In press 2010.

23. Saito S, Sakai M, Sasaki Y, Nakashima A, Shiozaki A. Inadequate tolerance induction may induce pre-eclampsia. J Reprod Immunol. 2007;76(1-2):30-9

24. Burton GJ, Charnock-Jones DS, Jauniaux E. Regulation of vascular growth and function in the human placenta. Reproduction. 2009; 138(6):895-902.

25. Cindrova-Davies T. Gabor Than Award Lecture 2008: pre-eclampsia - from placental oxidative stress to maternal endothelial dysfunction. Placenta. 2009;30 Suppl A:S55-65.

26. Redman CW, Sacks GP, Sargent IL. Preeclampsia: an excessive maternal inflammatory response to pregnancy. Am J Obstet Gynecol. 1999; 180(2 Pt 1):499-506.
27. Knight M, Redman CW, Linton EA, Sargent IL. Shedding of syncytiotrophoblast microvilli into the maternal circulation in pre-eclamptic pregnancies. Br J Obstet Gynaecol. 1998;105(6): 632-40.

28. Germain SJ, Sacks GP, Sooranna SR, Sargent IL, Redman CW. Systemic inflammatory priming in normal pregnancy and preeclampsia: the role of circulating syncytiotrophoblast microparticles. J Immunol. 2007; 178(9):5949-56.

29. Terrone DA, Rinehart BK, May WL, Moore A, Magann EF, Martin JN Jr. Leukocytosis is proportional to HELLP syndrome severity: evidence for an inflammatory form of preeclampsia. South Med J. 2000;93(8):768-71.

30. Derzsy Z, Prohászka Z, Rigó J Jr, Füst G, Molvarec A. Activation of the complement system in normal pregnancy and preeclampsia. Mol Immunol. 2010;47(7-8):1500-6.

31 . Borzychowski AM, Sargent IL, Redman CW. Inflammation and preeclampsia. Semin Fetal Neonatal Med. 2006;1 11(5):309-16.

32. Roberts JM, Taylor RN, Musci TJ, Rodgers GM, Hubel CA, McLaughlin MK. Preeclampsia: an endothelial cell disorder. Am J Obstet Gynecol. 1989;161 (5):1200-4.

33. Roberts JM. Endothelial dysfunction in preeclampsia. Semin Reprod Endocrinol. 1998; 16(1):5-15.

34. Redman CW, Sargent IL. Circulating microparticles in normal pregnancy and pre-eclampsia. Placenta. 2008;29 Suppl A:S73-7.

35. Cockell AP, Learmont JG, Smárason AK, Redman CW, Sargent IL, Poston L. Human placental syncytiotrophoblast microvillous membranes impair maternal vascular endothelial function. $\mathrm{Br} \mathrm{J}$ Obstet Gynaecol. 1997;104(2):235-40.

36. Maharaj AS, D'Amore PA. Roles for VEGF in the adult. Microvasc Res. 2007;74(2-3):100-13.

37. Maharaj AS, Walshe TE, Saint-Geniez M, Venkatesha S, Maldonado $A E$, Himes $N C$, et al. VEGF and TGF-beta are required for the maintenance of the choroid plexus and ependyma. J Exp Med. 2008;205(2):491-501.

38. Maynard SE, Min JY, Merchan J, Lim KH, Li J, Mondal S, et al. Excess placental soluble fms-like tyrosine kinase $1(\mathrm{sFl}+1)$ may contribute to endothelial dysfunction, hypertension, and proteinuria in preeclampsia. J Clin Invest. 2003; 111 (5):649-58.

39. Esser S, Wolburg K, Wolburg H, Breier G, Kurzchalia T, Risau W. Vascular endothelial growth factor induces endothelial fenestrations in vitro. J Cell Biol. 1998;140(4):947-59.

40. Levine RJ, Maynard SE, Qian C, Lim KH, England L, Yu KF, et al. Circulating angiogenic factors and the risk of preeclampsia. $\mathrm{N}$ Engl J Med. 2004;350(7):672-83.

41. Romero R, Nien JK, Espinoza J, Todem D, Fu W, Chung H, et al. A longitudinal study of angiogenic (placental growth factor) and anti-angiogenic (soluble endoglin and soluble vascular endothelial growth factor receptor-1) factors in normal pregnancy and patients destined to develop preeclampsia and deliver a small-for-gestational-age neonate. J Matern Fetal Neonatal Med. 2008;21(1):9-23.

42. Sebire NJ, Jolly M, Harris JP, Wadsworth J, Joffe M, Beard RW, et al. Maternal obesity and pregnancy outcome: a study of 287,213 pregnancies in London. Int J Obes Relat Metab Disord. $2001 ; 25(8): 1175-82$.

43. Duckitt K, Harrington D. Risk factors for pre-eclampsia at antenatal booking: systematic review of controlled studies. BM. 2005;330(7491):565.

44. Bianco AT, Smilen SW, Davis Y, Lopez S, Lapinski R, Lockwood CJ. Pregnancy outcome and weight gain recommendations for the morbidly obese woman. Obstet Gynecol. 1998;91(1):97-102. 
45. Pou KM, Massaro JM, Hoffmann U, Vasan RS, Maurovich-Horvat $P$, Larson $M G$, et al. Visceral and subcutaneous adipose tissue volumes are cross-sectionally related to markers of inflammation and oxidative stress: the Framingham Heart Study. Circulation. 2007; $116(11): 1234-41$.

46. Jarvie E, Hauguel-de-Mouzon S, Nelson SM, Sattar N, Catalano PM, Freeman DJ. Lipotoxicity in obese pregnancy and its potential role in adverse pregnancy outcome and obesity in the offspring. Clin Sci (Lond). 2010;1 19(3):123-9.

47. Erbay E, Babaev VR, Mayers JR, Makowski L, Charles KN, Snitow $M E$, et al. Reducing endoplasmic reticulum stress through a macrophage lipid chaperone alleviates atherosclerosis. Nat Med. 2009; 15(12):1383-91.

48. Gluckman PD, Hanson MA, Pinal C. The developmental origins of adult disease. Matern Child Nutr. 2005; 1(3):130-41.

49. Byers BD, Betancourt A, Lu F, Hankins GD, Longo M, Saade $G R$, et al. The effect of prepregnancy obesity and sFlt-1-induced preeclampsia-like syndrome on fetal programming of adult vascular function in a mouse model. Am J Obstet Gynecol. 2009;200(4):432.e1-7.

50. Amarilyo G, Oren A, Mimouni FB, Ochshorn Y, Deutsch V, Mandel D. Increased cord serum inflammatory markers in smallfor-gestational-age neonates. J Perinatol. 2011 ; 31(1):30-2.

51. Dong Y, Hou W, Wei J, Weiner CP. Chronic hypoxemia absent bacterial infection is one cause of the fetal inflammatory response syndrome (FIRS). Reprod Sci. 2009; 16(7):650-6.

52. Malaeb S, Dammann O. Fetal inflammatory response and brain injury in the preterm newborn. J Child Neurol. 2009;24(9): $1119-26$.

53. Guo R, Hou W, Dong Y, Yu Z, Stites J, Weiner CP. Brain injury caused by chronic fetal hypoxemia is mediated by inflammatory cascade activation. Reprod Sci. 2010;17(6):540-8.

54. Yinon Y, Kingdom JC, Odutayo A, Moineddin R, Drewlo S, Lai V, et al. Vascular dysfunction in women with a history of preeclampsia and intrauterine growth restriction: insights into future vascular risk. Circulation. 2010;122(18):1846-53. 